\title{
Detection of Known and Novel Viral Pathogens in Belgian Ixodes ricinus Ticks ${ }^{\dagger}$
}

\author{
Bert Vanmechelen ${ }^{1}$, Michelle Merino ${ }^{1}$, Valentijn Vergote ${ }^{1}$, Lies Laenen ${ }^{1}$, Ana Rita Lopes ${ }^{1}$, \\ Edwin Claerebout ${ }^{2}$ and Piet Maes ${ }^{1, *}$
}

1 Department of Microbiology, Immunology and Transplantation, Laboratory of Clinical and Epidemiological Virology, Rega Institute, KU Leuven, Herestraat 49/Box 1040, BE3000 Leuven, Belgium; bert.vanmechelen@kuleuven.be (B.V.); michelle.merino@student.kuleuven.be(M.M.); valentijn.vergote@kuleuven.be (V.V.); lies.laenen@kuleuven.be (L.L.); anarita.lopes@student.kuleuven.be(A.R.L.)

2 Faculty of Veterinary Medicine, Laboratory of Parasitology, Ghent University, Salisburylaan 133-D13, BE9820 Merelbeke, Belgium; edwin.claerebout@ugent.be

* Correspondence: piet.maes@kuleuven.be

† Presented at Viruses 2020-Novel Concepts in Virology, Barcelona, Spain, 5-7 February 2020.

Published: 16 June 2020

\begin{abstract}
Recent metagenomics studies have revealed several arthropod species to be major reservoirs for RNA viruses. One of these reservoirs is Ixodes ricinus, the most prevalent tick species in Europe, which is known to be a vector for many viral and bacterial pathogens. For this study, we decided to investigate the virosphere of Belgian Ixodes ricinus ticks. High-throughput sequencing of tick pools collected from six different sampling sites revealed the presence of viruses belonging to many different viral orders and families, including Mononegavirales, Bunyavirales, Partitiviridae, and Reoviridae. Of particular interest was the detection of several putative human pathogens, including members of the families Nairoviridae and Phenuiviridae as well as three new reoviruses, two of which cluster together with members of the genus Coltivirus. One of these two viruses represents a new strain of Eyach virus, a known causative agent of tick-borne encephalitis. All genome segments of this new strain are highly similar to those of previously published Eyach virus genomes, except for the fourth segment, encoding VP4, which is markedly more dissimilar, potentially indicating the occurrence of an antigenic shift. Further PCR-based screening of over 200 tick pools for 11 selected viruses showed that most viruses could be found in all six sampling sites, indicating the wide spread of these viruses throughout the Belgian tick population. Taken together, these results illustrate the role of ticks as important virus reservoirs, highlighting the need for adequate tick control measures.
\end{abstract}

Keywords: Ticks; Ixodes ricinus; Coltivirus

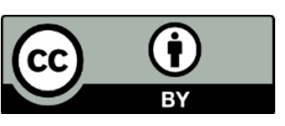

(C) 2020 by the authors. Licensee MDPI, Basel, Switzerland. This article is an open access article distributed under the terms and conditions of the Creative Commons Attribution (CC BY) license (http://creativecommons.org/licenses/by/4.0/). 